Graduate Thesis RePORTS

\title{
A pattern-based approach for developing creativity applications supported by surface computing
}

\author{
Yazmín Magallanes
}

Published: 21 September 2016

\begin{abstract}
Whereas creativity tasks have traditionally been supported by conventional media and tools, (such as paper, pens, scissors and glue), mobile phones, tablets and other devices based on interactive surfaces are increasingly been used as additional support. Large-sized multi-touch interactive surfaces appear as an interesting alternative for supporting collaborative creativity processes. However, they have mostly been used for visualization and navigation purposes. Their use as authoring means, which would be essential in creativity tasks, has only begun to be explored. We have identified a significant gap between the level of development tools and the abstractions required by end-user applications that aim to support creativity processes using interactive surfaces. Thus, we propose ISCALI (Innovation Solutions Centered on Activities for Large-sized Interfaces), a model that can be used both for describing and for prescribing the role of multi-touch surfaces in collaborative creativity tasks. Based upon ISCALI, we designed a general architecture for collaborative creativity applications; and we developed a set of building blocks, referred to as TOKAs (Touch Operations for Creative Activities). Applications that facilitate the use of various methodologies that foster collaborative creativity have been implemented by independent developers who have taken advantage of the availability of TOKAs. The implementation and use of TOKAs demonstrate ISCALI's expressivity for describing and guiding the development of applications that support collaborative creativity on top of interactive surfaces.
\end{abstract}

Keywords: Creativity work; multi-touch surfaces; Activity Centered Design; model; patterns; human-computer interaction; collaboration; interfaces; building blocks; cards; gestures.

\section{Research situation}

I am currently completing a $\mathrm{PhD}$ in Computer Science at UDLAP. My main research interests lie in the study of interfaces that foster creativity using multi-touch surfaces. During this semester I will present the defense of my thesis. Thus, I hope I get some ideas from this doctoral consortium.

\footnotetext{
Magallanes, $\mathrm{Y}$.

Interactive and Cooperative Technologies Lab

Universidad de las Américas Puebla (UDLAP)

Email: yazmin.magallanesvz@udlap.mx
}

\section{Context and motivation}

The work of creativity has to do with the generation of new ideas or new associations between ideas. Many techniques have been devised to facilitate the participation of individuals and groups in the work of creativity. We focus on the group dynamics that occur when participants share their ideas and select the one that is most appropriate to solve a problem, as in the case of the brainstorming method. Most of these techniques are carried out with the support of different tools and artifacts (such as pens, markers, paper, dice, or timers). More recently, some creativity techniques for idea generation have been supported by multitouch surfaces (MTS).

MTS are interfaces between humans and computers that provide many technological advantages. They are capable of identifying two or more points of contact between the user and the screen in different positions, offer direct-touch input and bimanual interaction in a natural way, enable simultaneous interaction between multiple users and have a higher level of input flexibility than a mouse. Thus, given their characteristics, MTS show great potential to support the work of creativity [1].

The success of software applications for MTS depends on how well they support human activities. Collaborative creative work involves generating a large number of idea representations in the form of verbal utterances, handwritten text, sketches, idea associations, diagrams and more elaborated narratives. If MTS are intended to effectively support groups involved in creative work, they must enable the diverse set of interactions and behaviors that take place in collaborative settings. Platforms for supporting a wide range of methodologies for fostering creativity are needed. Unfortunately, developing new MTS-based applications still requires significant effort in using low-level primitives that facilitate interaction with tactile surfaces and building complex components on top of them in order to implement natural user interfaces needed by creative workers.

We analyze the most relevant actions enabled in well-known projects that support idea generation using MTS. Table 1 shows some examples of these projects. Based upon this analysis of software development, we noted that the main actions performed during the sessions of creativity using MTS are creating cards (spaces for introducing ideas); writing ideas (representing ideas through images, text, free hand drawings and voice); deleting ideas (erasing unwanted ideas); linking ideas (defining associations among ideas); grouping ideas (categorizing ideas); ungrouping ideas (deleting categories); defining relationships 
(naming relations among ideas); and evaluating ideas (assigning value to previously generated ideas).

Table 1 Interaction patterns using MTS

\begin{tabular}{|c|c|c|c|c|c|c|c|c|c|}
\hline $\begin{array}{l}\text { Apps } \\
\text { designed } \\
\text { for } \\
\text { creativity } \\
\text { using MTS }\end{array}$ & 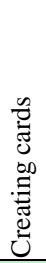 & 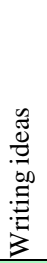 & 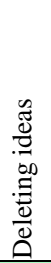 & 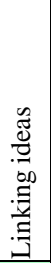 & 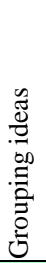 & 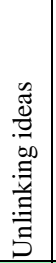 & 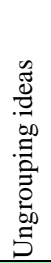 & 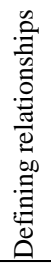 & 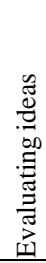 \\
\hline ScriptStorm [3] & $\mathrm{X}$ & $X$ & $X$ & $\mathrm{X}$ & $\mathrm{X}$ & $\mathrm{X}$ & $\mathrm{X}$ & NA & NA \\
\hline Trains of thought [6] & X & $X$ & NA & $X$ & $\mathrm{X}$ & $\mathrm{X}$ & $\mathrm{X}$ & NA & NA \\
\hline Affinity+ [2] & $\mathrm{X}$ & $\mathrm{X}$ & $\mathrm{X}$ & NA & $\mathrm{X}$ & NA & $\mathrm{X}$ & NA & NA \\
\hline Ideas Mapping [8] & $\mathrm{X}$ & $\mathrm{X}$ & $\mathrm{X}$ & $\mathrm{NA}$ & $\mathrm{X}$ & NA & $\mathrm{X}$ & NA & NA \\
\hline InnovIMM [7] & $X$ & $\mathrm{X}$ & $\mathrm{X}$ & $\mathrm{X}$ & $\mathrm{X}$ & $\mathrm{X}$ & $\mathrm{X}$ & $\mathrm{X}$ & NA \\
\hline $\begin{array}{l}\text { Generic creativity } \\
\text { techniques [4] }\end{array}$ & $\mathrm{X}$ & X & $\mathrm{X}$ & NA & $\mathrm{X}$ & NA & $\mathrm{X}$ & NA & $\mathrm{X}$ \\
\hline Reality-Based int [5] & $\mathrm{X}$ & $\mathrm{X}$ & $\mathrm{X}$ & $\mathrm{X}$ & $\mathrm{X}$ & $\mathrm{NA}$ & NA & $\mathrm{NA}$ & $\mathrm{NA}$ \\
\hline
\end{tabular}

\section{Research goals and methods}

The aim of this work is not only to motivate the use of MTS, but also to offer a new model for supporting the development of MTS-based creativity applications. In order to develop our conceptual model, we focus on finding interaction patterns with MTS during the creative process. We perform qualitative research to explore these interaction patterns and to achieve a detailed understanding of this issue. Our research methodology consists of the following steps:

1. Determine research questions

2. Design the study3. Collect data regarding interaction patterns during the creative process

3. Analyze creative methods

4. Generate a model

5. Pose a general architecture for developing applications

6. Validate the model

7. Report findings

\section{Dissertation status}

Understanding the context and the environment of the creative process (our research instrument) provides for better product development for these processes. Thereby our research questions arose: Is it possible to express the creative process in terms of ACD? And is it possible to help software developers in the construction of interfaces that support creativity?

We examined interaction patterns during the creative process in a conventional manner (utilizing pen and paper). And then, we examined the interaction patterns of people with MTS interfaces during these processes (our focus area). Characteristics that we sought in these samples were related to tools used and people's interaction with interfaces during the creative process.

For analysis of creativity methods we used ACD, as it focuses on understanding the actions and operations that make up activities in detail. Based on a thorough investigation of these actions and operations carried out by people in different processes of creativity using MTS, we obtained a set of interaction patterns. Thus, as part of this work we propose ISCALI, a model that can be used both for describing and for prescribing the role of multitouch surfaces in collaborative creativity tasks (see Figure 1).

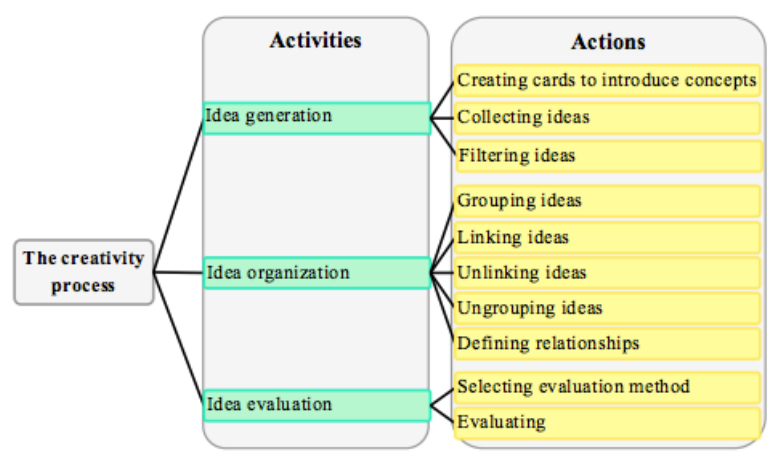

Figure 1. Creativity process using MTS and ACD model

Families of interaction patterns found during the processes that support creativity using MTS were "Creating cards to introduce concepts", "Collecting ideas", and "Filtering ideas" for idea generation activities; "Groping ideas", "Linking ideas", "Unlinking ideas", "Ungrouping ideas", and "Defining relationships" for idea organization activities; and "Selecting the evaluation method", and "Evaluating" for idea evaluation activities.

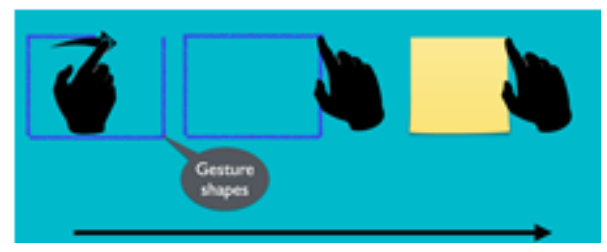

Figure 2. Creating cards by drawing a square

The act of creating cards (as for instance of a more detailed activity) consists of patterns to generate spaces where it is possible to represent ideas. Users can create a new card by: dragging and dropping a button, drawing a square (see Figure 2 as an example), bringing up a menu on any free space, using voice commands, using a physical object, using a menu associated with each object, and by tapping twice on an existing card.

Next, we designed an architecture (see Figure 3) that consists of three layers: the data layer, the services layer and the presentation layer. The data layer comprises the set of available interaction patterns. Part of our main contribution lies in linking this first layer to the next layer, the services layer, which comprises a set of building blocks. Here we conceptualize the interaction among the ISCALI components and the set of building blocks. Last but not least, the third layer is the presentation layer, which refers to the user- oriented functionality or where the user interaction with the system is managed.

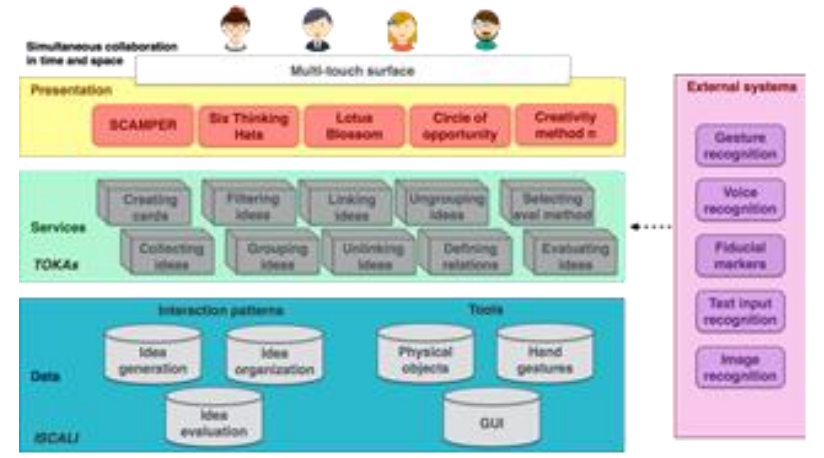

Figure 3. General architecture for developing applications for creativity 
In order to validate the model, we have implemented prototypical versions of these TOKAs and have made them available to independent developers. These developers have implemented applications that facilitate the use of various methodologies that foster collaborative creativity. Now we are designing experiments to observe users while conducting work that foment creativity. As a result of this, existing interaction patterns may be refined or additional interaction patterns might be derived.

\section{Results}

We have proposed ISCALI, a model that describes the main activities, actions and operations that are carried out by groups of innovators who use multi-touch surfaces as a part of the process of creativity. ISCALI is capable of expressing the main activities performed in salient projects that support the process of creativity using MTS. The implementation of TOKAs demonstrates ISCALI's expressivity for describing different mechanisms for stimulating creativity. Thus, key contributions of this research also include insights into the way in which a conceptual model (ISCALI) and a practical implementation of recurrent interaction patterns (TOKAs) can support the development of interfaces to foster creativity.

\section{References}

[1] Ardito, C. et al. 2015. Interaction with Large Displays: A Survey. ACM Comput. Surv. 47, 3 (Feb. 2015), 46:1-46:38.

[2] Burtner, E.R. et al. 2013. Affinity+: Semi-Structured Brainstorming on Large Displays. Technical Report
\#PNNL-SA-93014. Pacific Northwest National Laboratory (PNNL), Richland, WA (US).

[3] Clayphan, A. et al. 2014. ScriptStorm: Scripting to Enhance Tabletop Brainstorming. Personal Ubiquitous Comput. 18, 6 (Agosto 2014), 1433-1453.

[4] Frieß, M.R. et al. 2011. A Tabletop Application Environment for Generic Creativity Techniques. International Journal of Computer Information Systems and Industrial Management Applications (IJCISIM). 4, (2011), 55-65.

[5] Geyer, F. et al. 2011. Designing Reality-based Interfaces for Creative Group Work. Proceedings of the 8th ACM Conference on Creativity and Cognition (New York, NY, USA, 2011), 165-174.

[6] Jaco, A.A. et al. 2014. Trains of Thought on the Tabletop: Visualizing Association of Ideas Improves Creativity. Personal Ubiquitous Comput. 18, 5 (Jun. 2014), 1159-1167.

[7] Magallanes, Y. et al. 2012. Combining gestures and graphical elements for collaboration using multi-touch surfaces. (2012), 173-178.

[8] Zaphiris, P. et al. 2013. User Experience in Using Surface Computing for Collaborative Decision Making. Interactive Technology and Smart Education. 10, 4 (Nov. 2013), 297308. 